\title{
Lingua e identità nel carteggio degli intellettuali fiumani (1960-1987)
}

\author{
Corinna Gerbaz Giuliano, Maja Đurdulov
}

Il saggio prende in esame alcuni scambi epistolari intercorsi tra gli intellettuali fiumani Osvaldo Ramous, Antonio Widmar e Enrico Morovich dai quali si evince il rapporto con la loro identità. Questa è determinata dal singolare ambiente multiculturale e multilingue in cui sono nati e si sono formati. Il lavoro esamina le complessità che emergono dall' identità multiculturale degli autori fiumani di lingua italiana in un periodo di significative trasformazioni politiche, linguistiche e culturali a Fiume.

Parole chiave: circoli culturali a Fiume, lingua italiana, identità interculturale, Fiume

Članek obravnava korespondenco med reškimi intelektualci Osvaldom Ramousem, Antoniom Widmarjem in Enricom Morovichem, iz katere je razbrati odnos, ki ga imajo do svoje identitete. Njihova identiteta je namreč zaznamovana z medkulturnim in večjezičnim okoljem, v katerem so se rodili in odraščali. Delo analizira predvsem kompleksnost medkulturne identitete reških avtorjev italijanskega jezika v obdobju pomenljivih političnih, jezikovnih in kulturnih sprememb na Reki.

Ključne besede: kulturni krogi na Reki, italijanski jezik, medkulturna identiteta, Reka

Tercorsi culturali di Osvaldo Ramous, Antonio Widmar ed Enrico Morovich, voci illustri del cosiddetto circolo letterario fiumano, e le loro corrispondenze epistolari costituiscono una materia ancora per molti versi da studiare. Il presente contributo si soffermerà, in particolare, su ciò che emerge dal fitto carteggio tra $\mathrm{i}$ tre letterati e che attesta la preziosa eredità di un vivace mondo culturale che ha interessato la città di Fiume. Nella fattispecie si cercherà di mettere in rilievo la necessità, avvertita da Ramous, Widmar e Morovich, di conservare e archiviare il proprio registro linguistico e culturale, ricco e variegato. Per quanto concerne il discorso identitario, va sottolineato che il loro è stato un percorso piuttosto tormentato, simile e diverso al contempo, ma è proprio alla luce di questa considerazione che si è voluto indagare sulla sensibilità di questi intellettuali fiumani nei confron- ti del proprio nucleo identitario che emerge in particolare dalle loro corrispondenze private. Il processo di formazione dell'identità non è di pertinenza esclusiva delle discipline socio-antropologiche, ma implica indubbiamente il discorso letterario ed è in questa prospettiva che si è cercato di analizzare il senso di appartenenza all'entità collettiva dei tre autori fiumani filtrata da ciò che emerge dalle loro lettere.

Ramous, Widmar e Morovich nascono agli inizi del Novecento a Fiume, in una città di mare e di frontiera, che, al di là dell'elemento di instabilità racchiuso nell'accezione del termine, delinea una storia culturale ricchissima, fatta di rimandi, di compenetrazione culturale e linguistica.

Tutti e tre gli autori nascono in un ambiente pluriculturale e mistilingue e si inseriscono a pieno titolo nella koinè mitteleuropea, in quel cro- 
cevia di popoli e culture che funge da sostrato culturale alla città di Fiume. Il capoluogo quarnerino situato a ridosso del mare Adriatico può essere inteso come una sorta di collante che ha unito sia i litorali che gli entroterra ma, oltre ad unire, ha inevitabilmente prodotto scissioni tra $\mathrm{i}$ vari popoli dando vita a una storia molto movimentata, come lo è stata del resto tutta la storia dell'area balcanica

$$
\begin{aligned}
& \text { che è in gran parte la storia delle grandi po- } \\
& \text { tenze che si sono continuamente contese il } \\
& \text { dominio dei territori dell'odierna Repubbli- } \\
& \text { ca e hanno cercato di espandersi attraverso } \\
& \text { le sue strategiche vie d'accesso naturali. Ma è } \\
& \text { anche la storia della resistenza degli slavi del } \\
& \text { sud agli attacchi contro la loro indipenden- } \\
& \text { za e il loro carattere nazionale e, nello stesso } \\
& \text { tempo, della lotta di questi popoli per raffor- } \\
& \text { zare la loro posizione e dei tentativi degli uni } \\
& \text { di unificare, assorbire o dominare gli altri. }
\end{aligned}
$$

A prescindere dai pesanti momenti di instabilità $\mathrm{e}$ tensione che queste terre hanno vissuto, vanno ricordati i momenti di notevole crescita economico-sociale e culturale, basti ricordare il periodo della reggenza austro-ungarica, che in particolare per la città di Fiume ha significato diventare il secondo porto dell'Impero, incrementando così le attività commerciali e industriali, seguite da un considerevole progresso in chiave socio-culturale il che ha portato a consolidare l'aspetto multiculturale e in particolare di convivenza della città quarnerina. Una sorte analoga la ebbero anche i centri più rilevanti sia in terra istriana sia in quella dalmata, basti pensare alla Spalato di cui ci narra Enzo Bettiza. In questo quadro multietnico il concetto di cittadinanza a Fiume è ed è sempre stato sinonimo di apertura culturale e linguistica e soprattutto di tolleranza reciproca. Antonio Widmar, Osvaldo Ramous e Enrico Morovich sono intellettuali fiumani dal respiro cosmopolita, uomini di frontiera, personalità dall'identità plurima. Ci risulta indispen-

I Henry Clifford Darby, Robert William Seton-Watson, Phyllis Auty, Robert George Dalrympl Laffan e Stephen Clissold, Storia della Jugoslavia. Gli slavi del sud dalle origini a oggi (Torino: Einaudi, 1969), 13-14 sabile spendere alcune parole per tentare di circoscrivere (per quanto sia possibile) il concetto di identità plurima. Che cos'è in effetti l'identità plurima? Cerchiamo di spiegarlo affidandoci al saggio di Edmond Jabés Uno straniero con, sotto il braccio, un libro di piccolo formato, ${ }^{2}$ un libro autobiografico nel quale l'autore mette in scena il personaggio dello Straniero che si fonde con il narratore. Jabés in effetti racconta se stesso, è declinato alla prima persona. Chi è dunque lo Straniero di Jabés? È l'Io che incontra l'Altro, l'estraneo, il forestiero; è l' Io che incontra se stesso nelle sembianze di uno straniero. Dunque lo straniero siamo noi stessi. Sottolinea Pier Aldo Rovatti nella postfazione all'opera:

$$
\begin{aligned}
& \text { per Jabés si tratta, mediante un esercizio } \\
& \text { (che non può avere a che fare con la parola), } \\
& \text { cioè una ricerca e un travaglio, e in definitiva } \\
& \text { precisamente attraverso una messa a repen- } \\
& \text { taglio della propria soggettività, di 'diven- } \\
& \text { tare stranieri' [... . Jabés sa che questo Stra- } \\
& \text { niero, che noi vediamo solo di spalle, non è } \\
& \text { fuori di noi. Lo è solo nella finzione narra- } \\
& \text { tiva: per incamminarci davvero sulla strada } \\
& \text { dell'io-straniero si tratta certo di riconosce- } \\
& \text { re la passività-responsabilità dell'io, ma poi } \\
& \text { e prima di tutto si tratta di realizzare il fatto } \\
& \text { che quello Straniero che intravvedo e che mi } \\
& \text { sfugge sono io stesso. }
\end{aligned}
$$
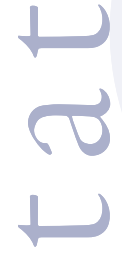

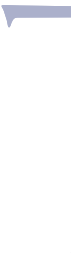

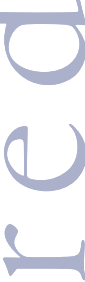

E per $\mathrm{i}$ tre autori in questione l'incontro dell'Io con se stesso nelle sembianze di uno Straniero rappresenta una condizione di partenza dalla quale si sviluppa la loro identità plurima che li fa diventare cittadini del mondo.

La città quarnerina, crogiuolo di etnie e culture diverse dai tempi più remoti, punta sulla formazione di un sostrato intellettuale multiculturale e plurilingue che si adopera nel manifestare la propria specificità, nel cancellare eventuali giudizi di parte dimostrando di meritare a pieno titolo il coinvolgimento negli aspetti culturali di mato (Milano: SE, I99I).

Pier Aldo Rovatti, "L'io straniero e il silenzio della parola", in Uno straniero con, sotto il braccio, un libro di piccolo formato, a cura di Edmond Jabès (Milano: SE, I991), I 40. 
rilievo. Dentro a questo quadro si muovono personaggi diversi tra di loro, ma concordi nell'affidare al discorso culturale la missione di costruire ponti tra culture differenti. Il ruolo degli intellettuali e dei mediatori culturali fiumani che si impegnano nell'avvicinare letterature diverse e si cimentano in traduzioni da una lingua all'altra, diviene un anello di congiunzione importante all'interno di un quadro pluriculturale, che di fatto incarna lo spirito della città quarnerina. Ed è proprio questa specificità del retroterra culturale fiumano a suscitare l'interesse degli studiosi ad ampia scala.

Sono molti i nomi di intellettuali fumani a cui va riconosciuto il merito di aver tessuto una così ricca storia culturale, tra questi spiccano quelli di Widmar, Ramous e Morovich. La scrittura è insieme la base della realtà sociale, della nostra coscienza e del nostro pensiero e la pratica letteraria si presta per definizione a funzione di archivio della memoria. Se siamo concordi nell'affermare che nella memoria di un popolo è intrinseco il concetto d'identità, allora le corrispondenze epistolari divengono materiale prezioso per indagare specifici periodi storici e forme di pensiero. All'interno della storia della gente di confine intessuta da rimandi, alti e bassi, scontri e conflitti, i carteggi diventano materiale pregiato per ricucire i lembi della storia con la $S$ maiuscola. I pensieri, gli stati d'animo e le posizioni a volte molto dure nei confronti di specifiche vicende storiche che emergono dai carteggi ci permettono di interpretare momenti storici particolari. Partendo dal presupposto che la memoria debba essere intesa come categoria indispensabile per capire il presente, allora il narrare allargato al discorso confidenziale delle lettere va inteso come parte integrante dell'esistenza umana che è «come la vita, esiste di per sé, è internazionale, trans-storica, trans-culturale $\gg .{ }^{4}$ La letteratura di confine e soprattutto i carteggi tra gli intellettuali costituiscono piattaforme per conoscere realtà diverse, proiezioni di vita da cui emergono valori, mentalità, culture a contatto

4 Roland Barthes, "Introduzione all'analisi strutturale dei racconti", in L'analisi del racconto (Milano: Bompiani, 1996), 7 e storie divise. Il terreno entro il quale gli intellettuali si muovono non è esclusivamente quello della memoria individuale, bensì è quello della memoria collettiva di un popolo. «Il fenomeno della memoria, nella molteplicità dei suoi aspetti, non è solo interdisciplinare, nel senso di non poter essere oggetto di una sola disciplina, ma anche controverso e contradditorio all' interno delle singole discipline» ${ }^{5}$ e le vicende storiche che hanno interessato Fiume, l'Istria e le isole, privandole in gran parte della componente italiana, sono divenute oggetto di riflessione letteraria, e non solo, in virtù del condizionamento reciproco che esiste tra letteratura e storia hanno fortemente delineato il discorso letterario.

Ma nel considerare il fenomeno della memoria collettiva nella sua diversità e contraddittorietà dobbiamo inevitabilmente fare i conti anche con la memoria del dolore. Sottolinea Nelida Milani che «l'identità personale è fondata sulla memoria, sulla propria autobiografia. Anche la sofferenza ha un ruolo nell'identità, un ruolo positivo» ${ }^{6}$ e la storia dell'area istro-quarnerina è fatta anche della memoria del dolore, è un valore del codice genetico dei suoi abitanti. «La memoria dà la capacità agli individui di aver coscienza di permanere se stessi attraverso il tempo e attraverso tutte le fratture dell'esperienza. Quindi l'identità personale viene connessa a una fondazione orizzontale, la quale mette in relazione gli istanti, le ore, i giorni della nostra esistenza con tutto l'arco della nostra vita organica. Quella della memoria collettiva è soltanto un'analogia».

La memoria rappresenta un terreno d'indagine di grandissima attualità. «Il terreno della memoria è dinamico, conflittuale, un luogo in cui i ricordi non giacciono statici in successione [...] ma sono legati ad affetti, appartenenze, giudizi, valori, interessi» ${ }^{8}$ e il carteggio, inteso quale

\footnotetext{
5 Aleida Assmann, Ricordare. Forme e mutamenti della memoria culturale (Bologna: Il Mulino, 2002), 17 .

6 Nelida Milani, "Appartenenza e separazione”, Civiltà istriana, ricerche eproposte. Etnia extra serie, n.I (1998): 289. Milani, “Appartenenza e separazione," 289.

8 Paolo Jedlowski, Il senso del passato. Per una sociologia della memoria (Milano: Franco Angeli, 1991), 9.
} 
parte integrante nella ricostruzione della memoria collettiva di un popolo, diventa un elemento di forza improsciugabile.

\section{Antonio Widmar}

Sono proprio gli affetti, i giudizi, le appartenenze e i valori che emergono dal carteggio tra Osvaldo Ramous e Antonio Widmar.

Antonio Widmar nasce a Fiume nel r899e muore a Chiavari nel 1980. Poeta, prosatore, pubblicista e traduttore. A conclusione degli studi letterari compiuti a Bologna, fa ritorno nella sua città. I primi vent'anni del secolo scorso lo vedono protagonista di interessanti iniziative culturali promosse dai periodici letterari "La Fiumanella" e "Delta". A partire dal 1926 ha inizio la sua parentesi ungherese; rimarrà a Budapest fino alla fine della seconda guerra mondiale, dove allaccerà rapporti d'amicizia con l'intellighenzia ungherese. Nel 1943 si rifiuterà di aderire alla Repubblica Sociale di Mussolini e nel 1944 sarà deportato assieme ad altri funzionari dell'ambasciata italiana in Germania. Ritornerà a Budapest e si lancerà nell'attività umanitaria, lavoro illegale che gli procurerà non pochi problemi. All'ingresso delle truppe sovietiche in Ungheria verrà arrestato e farà ritorno in Italia. Nel 1947 verrà nominato consigliere culturale presso l'Ambasciata italiana a Tokio, dove vi rimarrò fino al 1963. Seguirà un breve soggiorno diplomatico a Ginevra sino al 1964, anno del suo pensionamento.

Widmar e Ramous si incontrarono nel periodo in cui quest'ultimo si accostava al giornalismo, collaborando dal 1923 al 1925 alla rivista "Delta" di Fiume, diretta proprio dallo stesso Widmar, il quale la ritenne un po' la sua creatura visto che «in pieno fascismo era stata un'iniziativa di limpido carattere internazionalista, protesa a contribuire alla reciproca conoscenza tra $\mathrm{i}$

9 Corinna Gerbaz Giuliano, "Intorno agli scambi filosofici nel carteggio Ramous-Widmar," in Osvaldo Ramous. L'impegno culturale e critico, a cura di Gianna Mazzieri Sanković (Fiume: Edizione della Comunità degli Italiani di Fiume, 2008), 76-77. popoli e quindi alla loro autentica fratellanza», ${ }^{\circ \circ}$ e a "La Fiumanella", altra rivista importantissima, che segnò il percorso culturale di quel tempo. «La formazione di Widmar risentì fortemente del clima culturale fiumano degli anni Venti, di chiaro orientamento cosmopolita, proiettato ad operare una sorta di trait d'union tra il mondo slavo e le vicine culture mitteleuropee». ${ }^{\text {II }}$ Le posizioni espresse da Widmar tracciano un percorso critico in cui spiccano le sue convinzioni e idee politiche. In una lettera del 197I l'autore, dichiarando la sua posizione antidannunziana, fa emergere il senso dell'identità linguistica e culturale della Fiume dell'epoca e scrive:

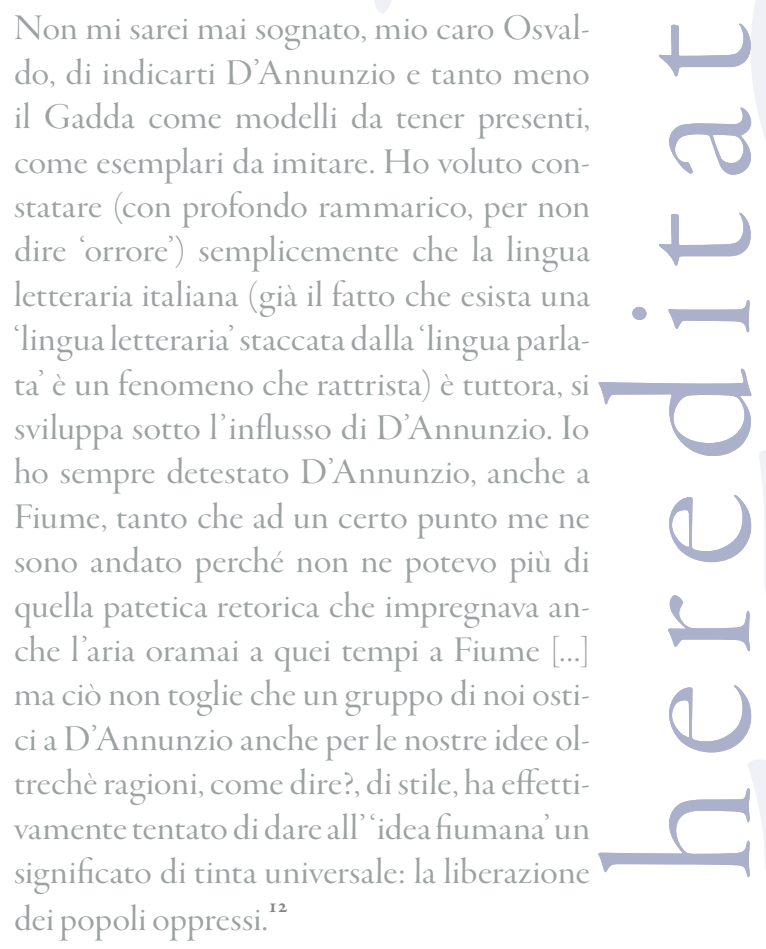

Il lettore viene colpito dalla dimensione intimistica che traspare dallo scambio epistolare tra i due amici: i temi delle lettere risultano variegati e le discussioni che si snodano tra Ramous

io Gianna Mazzieri-Sanković, "Osvaldo Ramous: un fiumano, cittadino del mondo," La Battana, n. speciale 2 (1997): 66.

I I Corinna Gerbaz Giuliano, "Antonio Widmar mediatore culturale e traduttore," Quaderni giuliani di storia, n. 36/I (2015): I25.

I2 Lettera di Antonio Widmar a Osvaldo Ramous, I3 aprile 197 I (archivio privato della famiglia), in Gerbaz Giuliano, "Antonio Widmar mediatore culturale e traduttore," I28. 
e Widmar riguardano questioni filosofiche, politiche e culturali. Al centro delle loro discussioni rimane sempre il discorso letterario che rappresenta per entrambi gli autori il fulcro della loro esistenza. Ciò di cui il lettore si accorge subito sono le dichiarazioni (spesso dirette e forti) e le prese di posizione che ne derivano. Così, in una lettera del 31 agosto 1965 , Ramous delinea all'amico Widmar il suo punto di vista circa la rivista "La Battana" che iniziò a uscire in quel periodo:

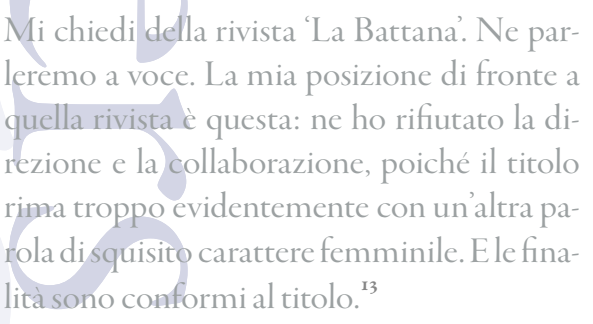

Antonio Widmar è altrettanto diretto nell'esprimere le proprie opinioni, il che si evince da una lettera successiva, datata 23 ottobre 1975 , in cui parla della rivista "Delta" e sottolinea:

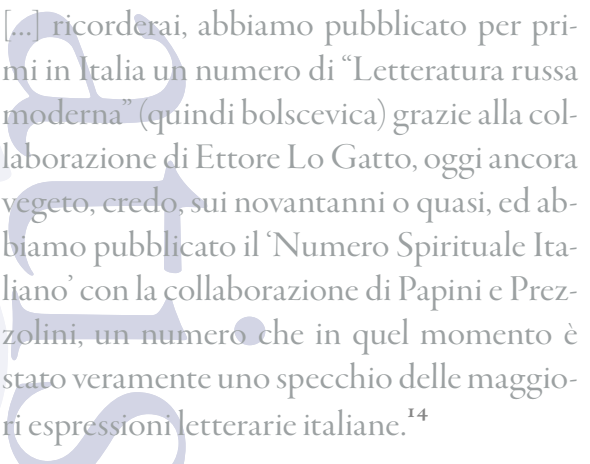

Anche in questo caso fa emergere quello spirito identitario che ha delineato la sua esistenza. Come molti altri fiumani della sua generazione, Widmar conosceva sicuramente quattro lingue: l'italiano, il croato, l'ungherese e il tedesco. Aveva frequentato le scuole ungheresi e, tra le altre sue attività, va sottolineata anche quella di traduttore. Fin da giovane iniziò a collaborare con gli ambienti culturali fiumani e, più tardi, con

I3 Lettera di Osvaldo Ramous ad Antonio Widmar, 3 I agosto 1965 (archivio privato della famiglia).

I4 Lettera di Antonio Widmar a Osvaldo Ramous, 23 ottobre 1975 (archivio privato della famiglia), in Gerbaz Giuliano, "Antonio Widmar mediatore culturale e traduttore," 128. quelli ungheresi e italiani. ${ }^{15}$ Questa attività culturale si affiancò a un certo punto della sua vita alla carriera diplomatica, ma entrambi questi indirizzi, non in contraddizione uno con l'altro, confluirono in ciò che lo rese un intellettuale cosmopolita, aperto a «una cultura che superasse i confini territoriali e linguistici ${ }^{16}{ }^{16}$

\section{Osvaldo Ramous}

Osvaldo Ramous, personalità di spicco dell'area istro-quarnerina, ha lasciato una traccia indelebile nella storia della letteratura fiumana.

\section{Ramous rappresenta la continuità di una let- teratura italiana autoctona dell' istroquarne- rino. La sua produzione letteraria è nata in un periodo in cui Fiume era parte dell'Un- gheria, si è sviluppata in direzione ermetica durante la Fiume italiana ma non ha smesso di esistere nemmeno negli anni successivi, con l'annessione della città alla Jugoslavia. ${ }^{17}$}

Il poligrafo fiumano ci ha lasciato un'eredità preziosa: undici libri di poesia, nove drammi dei quali cinque inediti, due raccolte di racconti, due romanzi, circa quattrocento tra articoli e saggi pubblicati su varie riviste, numerosi radiodrammi. Inoltre, ha firmato quarantasei regie del Dramma Italiano. «Innanzitutto poeta ma pure narratore, drammaturgo, giornalista, critico letterario e teatrale, traduttore, regista e direttore del Dramma Italiano, tradotto in tante lingue, conosciuto nell'America latina, vincitore di numerosi premi e riconoscimenti, Osvaldo Ramous rimane sconosciuto a molti». ${ }^{\text {r\$ }}$

Nell'Archivio di famiglia sono custoditi numerosi carteggi che forniscono una chiave di lettura particolare per ricostruire la sua personalità complessa e non solo letteraria. Nello scambio epistolare con diversi intellettuali appartenenti

is Gerbaz Giuliano, "Antonio Widmar mediatore culturale e traduttore," 125 .

I6 Gerbaz Giuliano, "Antonio Widmar mediatore culturale e traduttore," 129

I7 Corinna Gerbaz Giuliano e Gianna Mazzieri-Sanković, Non parto, non resto... I percorsi narrativi di Osvaldo Ramous e Marisa Madieri (Trieste: Deputazione di Storia Patria per la Venezia Giulia, 2013), 9.

I8 Gerbaz Giuliano e Mazzieri-Sanković, Non parto, non resto... I percorsi narrativi di Osvaldo Ramous e Marisa Madieri, 9. 
alla medesima area che hanno dovuto abbandonare il territorio in seguito alle vicende belliche, emergono il più delle volte pensieri intimi, dubbi e incertezze. Lo testimoniano in particolare lo scambio di lettere con Morovich e con Widmar, che non sono da considerarsi dei semplici carteggi, ma sono indubbiamente un qualcosa di più: sono memorie, racconti intimi, documenti fondamentali dai quali emergono momenti di storia vissuta, visioni personali e impronte culturali.

Ramous impersona una realtà specifica,
quella che segna l'esistenza degli italiani su
queste terre in un periodo tra i più travaglia-
ti, percorso da due guerre mondiali e per di
più in un ambiente di confine che vede al-
terato spesso il volto della propria città. Ep-
pure ama la propria città anche quando sen-
te per le strade una lingua che non conosce
e si ritiene sempre più 'esule su questa terra.
Un atteggiamento che diventa paradigma di
modernità e vede Ramous definirsi cittadi-
no del mondo in una comunione ideale con
altri autori del periodo non solo italiani e ju-
goslavi ma pure portoghesi, brasiliani e sve-
desi. ${ }^{19}$

Osvaldo Ramous viene considerato oggi il maggiore scrittore fiumano di lingua italiana. Nato a Fiume nel 1905 e scomparso nella stessa città nel $198 \mathrm{I}$, crebbe in una famiglia numerosa. A soli due anni rimase orfano di padre, di professione meccanico, e la madre accettò l'aiuto del fratello a sostenere la famiglia. Nonostante le modeste condizioni economiche della stessa, in casa ci fu una ricca biblioteca che permise al giovane Osvaldo di conoscere i classici della letteratura straniera e italiana, tra cui Shakespeare, Molière, Dante, Petrarca, Ariosto e Goldoni. Nonostante un'infanzia difficile, grazie all'aiuto dello zio il giovane Ramous poté frequentare gli studi presso la scuola Comunale, l'Istituto Tecnico Leonardo da Vinci e l'Istituto Magistrale Egisto Rossi, ma anche quelli musicali alla

19 Gerbaz Giuliano e Mazzieri-Sanković, Non parto, non resto... I percorsi narrativi di Osvaldo Ramous e Marisa Madieri, ı.
Scuola comunale di musica, dove seguì gli studi di violino e pianoforte. ${ }^{20}$

Ramos fu un uomo di cultura, capace di immergersi in tutti gli aspetti che riguardavano la cultura italiana dell'ambiente in cui operava, promuovendola al di fuori del suo ambiente e dialogando con altri intellettuali che avevano i suoi stessi interessi, nonostante facessero parte di lingue e culture diverse. Fu poeta, prosatore, drammaturgo, traduttore. Si accostò al giornalismo collaborando con le riviste letterarie fiumane di inizio secolo (come "La Fiumanella" e "Delta", ma estendendo ben presto il suo campo d'azione a numerose altre riviste della penisola), immergendosi in quel fervido e stimolante dibattito culturale che operava nella Fiume di quegli anni e che avrebbe continuato fino alla conclusione della seconda guerra mondiale. Dal '30 al ' 42 fu redattore della "Vedetta d'Italia" e dal 1944 direttore della stessa. Nel dopoguerra, dal 1946 al 196I, fu direttore del Dramma Italiano. Curò 46 regie, tradusse in italiano numerose opere della drammaturgia jugoslava, scrisse nove drammi e una decina di radiodrammi, $\mathrm{Fu}$ autore di dieci raccolte di poesie tra cui $\mathrm{Nel}$ canneto (1938), Vento sullo stagno (1953), Pianto vegetale (1967), La parola nel tempo (1969), Realtà dell'assurdo (1973), Pietà delle cose (1977). Sono suoi pure due romanzi, I gabbiani sul tetto e Il cavallo di cartapesta, numerosi racconti, pubblicati in parte su riviste italiane e in parte confluiti nel volume Lotta con l'ombra e altri racconti (2006) e circa quattrocento articoli e saggi letterari pubblicati su riviste italiane, jugoslave, americane e francesi. ${ }^{2}$

Fiume nel secondo dopoguerra mutò drasticamente nella sua essenza: l'assetto politico e, di conseguenza, quello demografico e sociale risultarono ancora una volta, nel giro di qualche decennio, trasformati. Ramous, in questo nuovo contesto, continuò il suo operato culturale di tipo cosmopolita, di cittadino del mondo, atto a oltrepassare i confini laddove questi furono im-

\footnotetext{
20 Gerbaz Giuliano e Mazzieri-Sanković, Non parto, non resto... I percorsi narrativi di Osvaldo Ramous e Marisa Madieri, 47-48.

2 I Gianna Mazzieri, La Voce di una minoranza (Torino: La Rosa, 1998) 40.
} 
posti. Continuò così a tessere una rete di rapporti culturali che confluirono in collaborazioni e amicizie con numerosi intellettuali dell'epoca. Queste relazioni trovano la loro maggiore espressione nel ricco epistolario ramousiano presente nel lascito dell'autore. Gli intellettuali italiani e jugoslavi con i quali Ramous dialogava venivano coinvolti in discussioni riguardanti le loro ultime pubblicazioni, le possibili traduzioni reciproche, ma anche temi filosofici come la condizione dell'intellettuale e dell'uomo in genere. In questo senso sono significative alcune lettere che Ramous scrisse nella seconda metà degli anni 'so in cui denunciava apertamente le condizioni in cui si trovava a operare un autore il quale, trovandosi tra due nazioni il cui confine era stato stabilito da poco, non riusciva a esprimere fino in fondo il proprio potenziale. Così, in una lettera del I955 allo scrittore Mirko Božić rivela:

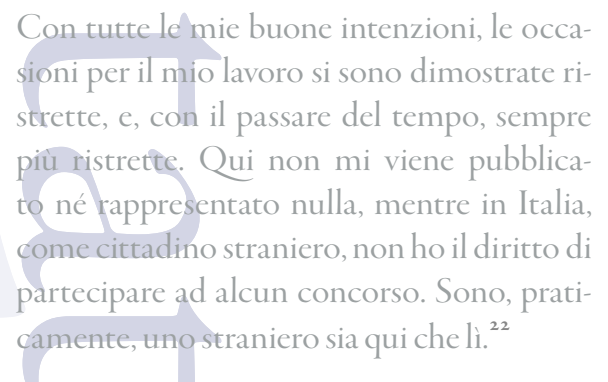

Ramous si sente straniero sia in Jugoslavia che in Italia. Nonostante abbia sempre scritto le sue opere in italiano, la lingua utilizzata non gli apre le porte del mercato editoriale in Italia. Lo stesso problema persiste anche in Jugoslavia, perché Fiume, dal secondo dopoguerra, ha come lingua ufficiale una lingua che non è l'italiano. Tutto ciò avveniva nonostante il fatto che Ramous avesse sempre cercato di mantenere vivo il dialogo tra scrittori italiani e jugoslavi. Prova ne sono le lettere con gli intellettuali jugoslavi e italiani dell'epoca (Drago Ivanišević, Augustin Stipčević, Eraldo Miscia, Ante Cettineo, Enrico Morovich, Antonio Widmar, Ugo Longo, Giacomo Oreglia, Andrè Charmel, Bino Rebellato

22 Ramous Osvaldo, lettera a Mirko Božić, 8 settembre 1955, custodita nell'archivio di famiglia e riportata in Gianna Mazzieri-Sanković e Maja Đurđulov, "Intorno agli scambi epistolari di Osvaldo Ramous," Quadernigiuliani distoria, n. 36/I (2015): I 17. ecc.) attraverso le quali cercava di far dialogare i due mondi culturali.

Una delle iniziative di Ramous che rientrano in questo programma è la pubblicazione nel 1959 del volume Poesia jugoslava contemporanea. Prima traduzione in lingua italiana che, in un periodo in cui la poesia jugoslava era quasi sconosciuta nella penisola, fu una delle prime antologie del genere in Italia. ${ }^{23}$ Qualche anno più tardi, nel 1964, organizzò a Cittadella il primo incontro tra scrittori italiani e jugoslavi.

Nonostante il suo impegno per promuovere il dialogo culturale tra le due sponde dell'Adriatico, Ramous incontrò nel suo percorso non pochi ostacoli, provenienti addirittura da qualche intellettuale della sua città. $\mathrm{Al} 2^{\circ}$ Festival della poesia jugoslava, organizzato a Fiume nel 1958, Ramous non venne invitato per cui denuncia il fatto in una lettera del 5 giugno 1958 indirizzata all'Associazione dei letterati della Croazia e al Consiglio dei letterati della Jugoslavija a Belgrado:

$$
\begin{aligned}
& \text { Al festival della poesia jugoslava, tenutosi a } \\
& \text { Fiume dal } 3 \text { I maggio al } 5 \text { giugno, non sono } \\
& \text { stato invitato né come poeta, né come tra- } \\
& \text { duttore, né come organizzatore, né in al- } \\
& \text { cun altra funzione. [...] Sono venuto a cono- } \\
& \text { scenza (e sono autorizzato a riportarlo) del } \\
& \text { fatto che durante una riunione, svoltasi a Za- } \\
& \text { gabria tra i membri della giuria del festival e } \\
& \text { alcuni rappresentanti dell'ambito educati- } \\
& \text { vo di Fiume, sia stato previsto il mio invito } \\
& \text { al Festival, al quale, però, si è opposto il pre- } \\
& \text { sidente del Consiglio per la cultura del cir- } \\
& \text { condario fiumano (direttore, tra l'altro, del } \\
& \text { Novi list') Milan Slani, dichiarando che tale } \\
& \text { invito sarebbe stato discusso a Fiume a causa } \\
& \text { di questioni specifiche di questa città'. [...] La } \\
& \text { mia esclusione dal festival ha una connota- } \\
& \text { zione molto negativa se si prende in conside- } \\
& \text { razione il fatto che al festival sia stato invita- } \\
& \text { to anche il poeta Luigi Fiorentino, direttore }
\end{aligned}
$$

23 Elvio Guagnini, "Osvaldo Ramous mediatore tra culture. Il critico e il giornalista," in Osvaldo Ramous. L'impegno culturale e critico, a cura di Gianna Mazzieri Sanković (Fiume: Edizione della Comunità degli Italiani di Fiume, 2008), 23. 


\begin{abstract}
della rivista 'Ausonia' (di Siena), alla quale collaboro. Fiorentino, naturalmente, non ha potuto non notare che - mentre lui stesso è un italiano residente in Italia invitato al festival - l'unico autore della minoranza italiana, invece, che abita a Fiume, è stato escluso dal festival. [...] Questo atteggiamento nei miei confronti mi colpisce ancora di più se penso a quanti anni ho sacrificato per dedicare una buona parte del mio impegno letterario alla traduzione e alla diffusione della letteratura jugoslava all'estero. [...] In questi giorni ho terminato un'antologia di poesia jugoslava contemporanea ( 52 autori, 86 poesie), che verrebbe stampata quest'anno da Dino Rebellato, editore di Padova. Per questa, come anche per le traduzioni degli altri autori jugoslavi, stampate all'estero, non ho mai cercato né riceverò mai alcun compenso. ${ }^{24}$
\end{abstract}

Nella lettera emerge chiaramente la delusione dell'intellettuale che si impegna fortemente per il benessere della propria città, cercando di promuovere la collaborazione tra culture diverse e di mantenere a Fiume un pluralismo linguistico, letterario e culturale, ma al quale questo tipo

24 »Na II. jugoslavenski festival poezije, koji se je održao u Rijeci od 3I.V. do 5.VI., ja nisam bio pozvan ni kao pjesnik, ni kao prevodilac, ni kao organizator, ni u bilo kojem drugom svojstvu. (...) Saznao sam (a ovlašten sam da to i saopćim), da je na jednom sastanku, održanom u Zagrebu izmedu književnika festivalskog žirija i nekih predstavnika prosvjete iz Rijeke, bilo predvideno da i ja budem pozvan na Festival, ali da se je tome suprotstavio predsjednik Savjeta za kulturu kotara Rijeka (koji je ujedno i direktor riječkog „Novog lista”) Milan Slani, izjavivši da će se taj moj poziv prodiskutirati u Rijeci radi 'specifičnih prilika ovoga grada'. Moje isključenje iz festivala ima vrlo neugodan značaj ako se uzme u obzir da je na taj festival pozvan i pjesnik Luigi Fiorentino, direktor revije "Ausonia” (iz Siene) u kojoj ja suradujem. Fiorentino, naravno, nije mogao a da ne primjeti da - dok je on, Talijan iz Italije primio poziv- dotle je jedini pjesnik talijanske manjine, koji živi u Rijeci, iskljućen iz tog festivala. (...) Ovaj stav prema meni pogada me tim gorče kad pomislim koliko sam svojih godina žrtvovao posvetivši dobar dio svoga književnoga rada prijevodima i difuziji jugoslavenske literature u inozemstvu (...), a ovih dana upravo sam završio rad na jednoj antologiji suvremene jugoslavenske poezije ( 52 pjesnika, 86 pjesama), koju bi štampao ove godine Bino Rebellato, nakladnik iz Padove. Za taj, kao i za ostale prijevode jugoslavenskih pjesnika, štampane u inozemstvu, niti sam tražio niti ću primiti nikakva honorara «. Ramous Osvaldo, 8 settembre 1955, lettera custodita nell'archivio di famiglia e riportata in Gianna Mazzieri-Sanković e Maja Đurđulov, "Intorno agli scambi epistolari di Osvaldo Ramous," I 16 (traduzione delle autrici) di attività, per svariati motivi, viene spesso ostacolata.

\section{Enrico Morovich}

Nel ricco epistolario ramousiano troviamo testimonianze anche di chi sta dall'altra parte, di chi ha deciso di intraprendere la sofferta via dell'esodo da Fiume. Tra questi c'è Enrico Morovich, scrittore fiumano nato nel 1906 e stabilitosi a Genova nel 1958 dopo un periodo di spostamenti lungo la penisola italica. Come riportato nella nota biografica curata da Marina Petronio, ${ }^{25}$ Morovich a diciott'anni conseguì il diploma di ragioniere. Nonostante maturasse nell'intimo la vocazione dello scrittore, fu impiegato presso la Banca d'Italia prima e i Magazzini Generali di Fiume poi. Nel 1929 iniziò la collaborazione con le riviste "La fiera letteraria" e "Solaria". Nel I950 lasciò Fiume e visse prima a Napoli, poi a Busalla, Lugo, Pisa e nel 1958 prese dimora fissa a Genova, dove lavorò presso il consorzio portuale. Collaborò con riviste e giornali importanti, come "Il Mondo", "La Nazione”, "Il Caffè, "Il Giornale d'Italia” ecc. La sua produzione comprende raccolte di racconti (L'osteria sul torrente, Miracoli quotidiani, I ritratti del bosco, Nostalgia del mare, Ascensori invisibili, Racconti di Fiume e altre cose, Notti con la luna), romanzi (Non era bene morire, Contadini sui monti, Il baratro, Piccoli amanti, finalista al Premio Strega, La caricatura, I giganti marini) e poesie (Cronache vicine elontane).

Nella corrispondenza tra Morovich e Ramous, il primo confessa all'amico alcune delle difficoltà che pure lui trova nell'ambito editoriale italiano, come fa Ramous nella già citata lettera a Mirko Božić. Morovich, tuttavia, in questa situazione non auspica una sorte diversa di quella che gli è toccata ma attribuisce proprio al suo percorso fiumano il merito per la sua propensione alla scrittura:

\footnotetext{
Sono escluso da un' importante antologia di Solaria, ma non credere ch'io abbia prote-

25 Marina Petronio, "Enrico Morovich: cenni biografici" in L'ultimo sapore della vigna, a cura di Enrico Morovich, (Trieste: LINT, 2002), 199-20I.
} 
stato o che mi sia lamentato da qualche par-

te. Sto bene cosi e non mi sento affatto ab-

bandonato ed occulto, penso piuttosto che

se avessi avuto la loro fortuna o il destino di

cominciare a lavorare a Genova invece che a

Fiume, non avrei mai scritto nulla, sarei ma-

gari diventato agente di borsa [... ${ }^{26}$

Per Morovich la nascita e la vita a Fiume rappresentarono le condizioni necessarie per essere divenuto quello che era: un artista, uno scrittore la cui città natale ha saputo innestare in lui lo spirito dell'espressione creativa. Una città che ha subito determinati processi politici e culturali importanti nel corso del Novecento e che, in tutto questo tempo, fu impregnata da quello che Marina Petronio definisce «fervore letterario cosmopolita $\gg .{ }^{27}$ La stessa studiosa nota come Morovich ne sia uno degli esempi caratterizzanti: in casa sua, «oltre alla conoscenza della madrelingua, erano di casa il tedesco e l'ungherese». ${ }^{28}$ Inoltre, Fiume fu, non a caso, la città di numerosi germanisti e studiosi di lingua e cultura ungherese. È proprio da Fiume che, tra il XIX e il XX secolo, partirono le prime traduzioni in lingua italiana di testi letterari ungheresi. Gli intellettuali fiumani, perfettamente bilingui ed esperti della cultura ungherese, avviarono un processo di scambi culturali: le prime traduzioni erano destinate al pubblico fiumano ma, successivamente, si iniziò a tradurre anche per le grandi case editrici italiane. È proprio così che attività del genere, che erano state avviate a finalità didattiche, finirono per espandersi su larga scala creando riviste culturali e letterarie e dando vita a collane di traduzione di opere ungheresi presso i più rinomati editori italiani. Alcuni degli intellettuali fiumani che fecero parte della generazione che avviò questo genere di traduzioni dall'ungherese furono Enrico Burich, Silvino Gigante, ma anche altri, come Antonio Widmar e Paolo

26 Morovich Enrico, lettera a Osvaldo Ramous, 8 agosto 1975, riportata in Gianna Mazzieri-Sanković, "Lettere fiumane. Morovich e Ramous: due scelte," Archeografo Triestino, s. IV v. LXVIII. (2008): 230.

27 Marina Petronio, "Premessa", in L'ultimo sapore della vigna, a cura di Enrico Morovich, (Trieste: LINT, 2002), 6

28 Marina Petronio, "Premessa", 6.
Santarcangeli, che continuarono la loro opera di mediazione culturale anche dopo aver lasciato Fiume. Tra gli autori ungheresi più tradotti troviamo molti scrittori rinomati, autori di veri capolavori, come Ferenc Molnár e Ferenc Herczeg, i quali, grazie al successo che ottennero presso il pubblico italiano, aprirono le porte in Italia alle traduzioni di quegli autori che la critica considera come i più importanti della letteratura ungherese moderna. ${ }^{2}$

Fiume fu indubbiamente un crocevia di culture e lingue, ma lo fu pure la vicina Sussak. Di quest'ultima viene spesso sottaciuto lo spirito altrettanto cosmopolita. In una lettera del 1984 indirizzata a Rinaldo Derossi, Morovich sottolinea la multiculturalità di Sussak:

\section{Era una piccola Babilonia come Fiume. Fu lì che da ragazzino imparai a scrivere tutti i cognomi fossero polacchi o boemi e che ve- devo subito passato il ponte entrando a Sus- sak nomi di bottegai come Angelini, Turina e cosi via. ${ }^{30}$}

In un'altra lettera, Morovich racconta la situazione linguistica della sua famiglia:

In casa quando eravamo bambini le zie sorelle del babbo ci parlavano il tedesco e siccome in casa tutti parlavano il fiumano eravamo abituati al bilinguismo.

Per tacere il piccolo dramma dell' iscrizione nelle scuole ungheresi di Fiume nel I9I3. Infatti una zia che insegnava nelle scuole ungheresi, intuì che avrei imparato anche l'ungherese con facilità [.... In casa c'erano delle riviste in lingua ungherese, ma non ricordo d'aver visto alcun libro illustrato. ${ }^{3 \mathrm{I}}$

Ciò che rimane a Morovich, dopo una vita vissuta a Fiume e un'altra vissuta fuori da Fiume, sono anche i ricordi. Ricordi che riaffiorano a Genova, città per molti versi simile alla sua cit-

29 Peter Sárközy, "Le traduzioni italiane delle opere letterarie ungheresi," Rivista distudi ungheresi, ns., n.3 (2004), 7-10.

30 Morovich Enrico, lettera a Rinaldo Derossi, 15 maggio 1984, in Morovich, L'ultimo sapore della vigna, 54

31 Morovich Enrico, lettera a Rinaldo Derossi, 22 luglio 1987, in Morovich, L'ultimo sapore della vigna, 116. 
tà natale, la quale mantiene le sue radici sempre salde nello scrittore. Questo ha spesso ribadito il suo sentirsi a proprio agio anche fuori da Fiume:

\section{Penso che di tanto in tanto sarebbe bene parlare magari un po' della nostra vita di esu- li in Italia. Nessuno può liberarsi delle pro- prie radici, ma pure, tante volte qui, quanto a Napoli, in Romagna e in Toscana mi sono sentito perfettamente a mio agio e in certo senso anche felice. ${ }^{32}$}

Ma sono proprio le radici che rimangono in Morovich, quelle radici che non si possono estirpare e rimangono nell'individuo ovunque lui vada. Sono radici soprattutto culturali le quali, più che attaccarsi all'individuo, sono origini a cui l'individuo si attacca:

Sono commosso. E rido. Di me, di noi che se anche ci trasferissimo a Parigi, dove senza imbarazzo finiremmo per parlare francese e anche per scriverlo, resteremmo sempre gente delle nostre parti.

I sogni mi dicono che le radici per quanto ci si allontani, restano sempre attaccate. Ossia noi attaccati alle radici. In sogno mi sono trovato anche in America. Ma senza farci gran caso. Una volta annotavo i miei sogni. Ora non più.

I buoni sono indimenticabili, gli altri svaniscano pure. ${ }^{33}$

\section{Conclusione}

Personaggi del calibro di Widmar, Ramous e Morovich sono nati e cresciuti in un contesto che non solo era plurilingue, ma era il punto d'incontro e di passaggio di altrettante culture. È significativo che essi abbiano poi intrapreso dei percorsi personali che, sebbene molto diversi tra di loro, hanno una matrice in comune, e cioè l'impegno di tipo interculturale, che ha

32 Morovich Enrico, lettera a Rinaldo Derossi, senza data, in Morovich, L'ultimo sapore della vigna, I6.

33 Morovich Enrico, lettera a Rinaldo Derossi, 27 marzo 1987, in Morovich, L'ultimo sapore della vigna, 108. mantenuto sempre salde le loro radici identitarie. Nonostante avessero operato in modi diversi e spesso contrastanti uno rispetto all'altro, e di conseguenza avessero avuto sorti di vita differenti, questo contributo ha voluto dimostrare come ci siano dei motivi che si ripetono e ritornano in ciascuna delle storie raccontate.

Il modello culturale che Fiume ha offerto loro e l'attaccamento per questa città hanno influito fortemente sul loro operato, il quale trova espressione nelle loro opere e nella corrispondenza personale che ci è disponibile. Questa espressione è diversa da caso a caso e dipende in parte dalle differenti vicende biografiche. In Widmår è evidente la curiosità per le diverse culture compresenti in lui, in primis quella italiana e quella ungherese, ma anche l'apertura a nuovi mondi e spazi, visibile anche, per esempio, nell'interesse per la cultura giapponese, coltivato durante gli anni di permanenza in Giappone come diplomatico dell'ambasciata italiana.

Anche in Ramous sono presenti questi tratti, nonostante egli avesse avuto una sorte ben dissimile da quella di Widmar e da Morovich, essendo l'unico dei tre a essere rimasto a Fiume per tutta la vita. Anche se Fiume durante la sua vita aveva cambiato diverse volte lingua e nazionalità, egli si assunse il compito di far dialogare i diversi mondi che vi transitavano e si compenetravano.

Scrivendo a proposito delle riviste fiumane degli anni '20, "La Fiumanella" e "Delta”, Ervin Dubrović sottolinea:

Gli intellettuali raccolti attorno a questi pe riodici erano di cultura italiana, ma, passata la sbornia dovuta alla pronunciata ostentazione d'italianità, essi avevano acquisito un orientamento cosmopolita essendo perfettamente consci della posizione culturale di Fiume, trait d'union tra il mondo limitrofo slavo e le vicine culture mitteleuropee, ri conoscendo alla propria missione il ruolo di intermediaria fra dette culture per cui aspiravano a far conoscere agli italiani la cultu- 
ra slava e agli slavi la letteratura e l'arte italiane

Ciò che accomuna, però, tutti questi personaggi è la loro città natale, perché è essa che, in fondo, è il comune denominatore tra tutti loro. Fiume si delinea, così, con le sue molteplici culture e lingue, come punto d'incontro tra biografie, storie e destini diversi, addirittura contrastanti tra di loro, ma pienamente unitari nella volontà di salvaguardare e ricordare la preziosa identità di questa città.

\section{Povzetek}

Članek obravnava pisma reških intelektualcev, kot so bili Osvaldo Ramous, Antonio Widmar in Enrico Morovich, ki še najprej dokazujejo njihov odnos do identitete, ki jo je oblikovalo enkratno okolje, v katerem so odraščali in bili vzgojeni. Specifika Reke na začetku 20. stoletja je bila namreč večkulturnost mesta, ki je imelo dolgo tradicijo večjezičnosti in je v nekaj desetletjih kar petkrat zamenjala politično upravo.

Omenjeni avtorji izražajo svojo večkulturnost na različne načine. Iz njihovih pisem drugim avtorjem, novinarjem in publicistom izhajajo namreč zanimive tematike, kot so lahko položaj pisatelja v novih političnih razmerah in splošni stiki med raznimi kulturami, s katerimi prihajajo vsakodnevno v stik tako v literarnem kot splošno družbenem življenju.

Članek vsebuje tako abstraktna (filozofske in jezikovne) kot praktična (zgodovinske in politične) razmišljanja, ki izhajajo iz pisem prej omenjenih avtorjev, in dokazujejo predvsem identifikacijo identitete s kompleksnim jezikovnim, političnim in kulturnim okoljem. Cilj raziskave je analizirati kompleksne tematike, ki so vzklile iz večkulturne identitete reških intelektualcev, ki so uporabljali italijanščino v času pomembnih političnih, jezikovnih in kulturnih sprememb na Reki, - a tudi v Evropi - in so poskušali definirati bistvo svoje identitete.

Kljucne besede: kulturni krogi na Reki, italijanski jezik, medkulturna identiteta, Reka

\section{Summary}

This paper analyses the letters of intellectuals from $\mathrm{Ri}$ jeka, such as Osvaldo Ramous, Antonio Widmar, En- rico Morovich, which demonstrate their relationship to identity, determined primarily by the unique environment in which they grew up and gained their education. The specificity of Rijeka at the beginning of the 2oth century is the multiculturalism of a city that has a long tradition of multilingualism and in just a few decades changes five times the political system.

These authors express their multiculturalism in different ways. From their mutual letters, and letters to other writers, journalists and publishers, derive interesting topics, such as the position of the writer in the new political environment and the general connections between various cultures with which they relate in their literary activities and in everyday life.

This paper presents some abstract (philosophical and linguistic) and practical (historical and political) considerations which can be read from the letters of the aforementioned intellectuals and which are based on the identification of the identity with a complex linguistic, political and cultural environment. The aim of this study is to analyse the complex issues arising from the multicultural identity of Rijeka's writers who used Italian in the years of important political, linguistic and cultural changes - in Rijeka, but also in Europe - and were trying to define the core of their identity.

Keywords: cultural circles in Rijeka, Italian language, multicultural identity, Rijeka

\section{Bibliografia}

Assmann, Aleida. Ricordare. Forme e mutamenti della memoria culturale. Bologna: Il Mulino, 2002.

Barthes, Roland. "Introduzione all'analisi strutturale dei racconti.” In L'analisi del racconto, 5-46. Milano: Bompiani, 1996.

Darby, Henry Clifford, Seton-Watson, Robert William, Auty, Phyllis, Laffan, Robert George Dalrympl, e Clissold Stephen. Storia della Jugoslavia. Gli slavi del sud dalle origini a oggi. Torino: Einaudi, 1969.

Dubrović, Ervin. "Francesco Drenig. Personaggio d'avanguardia e guida spirituale dei giovani." La Battana, n. speciale 2. (1997): 33-36.

Gerbaz Giuliano, Corinna. "Antonio Widmar mediatore culturale e traduttore." 
Quaderni giuliani di storia, n. 36/1. (2015): I2I-I29.

Gerbaz Giuliano, Corinna. "Intorno agli scambi filosofici nel carteggio RamousWidmar." In Osvaldo Ramous. L'impegno culturale e critico, a cura di Gianna Mazzieri Sanković, 75-87. Fiume: Edizione della Comunità degli Italiani di Fiume, 2008.

Gerbaz Giuliano, Corinna e MazzieriSanković, Gianna. Non parto, non resto... I percorsi narrativi di Osvaldo Ramous e Storia Patria per la Venezia Giulia, 2013.

Guagnini, Elvio. “Osvaldo Ramous mediatore tra culture. Il critico e il giornalista." In Osvaldo Ramous. L'impegno culturale e critico, a cura di Gianna Mazzieri Sanković, 23-34. Fiume: Edizione della Comunità degli Italiani di Fiume, 2008.

Jabès, Edmond. Uno straniero con, sotto il braccio, un libro di piccolo formato. Milano: SE, I99I.

Jedlowski, Paolo. Il senso del passato. Per una sociologia della memoria. Milano: Franco Angeli, I99I.

Mazzieri-Sanković, Gianna. "Lettere fiumane. Morovich e Ramous: due scelte." Archeografo Triestino, s. IV v. LXVIII. (2008): 227-239.

Mazzieri, Gianna. La Voce di una minoranza. Torino: La Rosa, 1998.

Mazzieri-Sanković, Gianna. "Osvaldo Ramous: un fiumano, cittadino del mondo." $\mathrm{La}$ Battana, n. speciale 2. (1997): 66-73.

Mazzieri-Sanković, Gianna, Đurđulov, Maja. "Intorno agli scambi epistolari di Osvaldo Ramous." Quaderni giuliani di storia, n. 36/I. (2015): IOI-I2O.

Milani, Nelida. "Appartenenza e separazione.” Civiltà istriana, ricerche e proposte. Etnia extra serie, n.I (1998): 289-310.

Morovich, Enrico. L'ultimo sapore della vigna. Trieste: LINT, 2002.

Morovich, Enrico. Non era bene morire.

Milano: Rusconi, 1992.

Rovatti, Pier Aldo. "L'io straniero e il silenzio della parola”. In Uno straniero con, sotto il braccio, un libro di piccolo formato, a cura di Jabès Edmond. Milano: SE, I99I.

Sárközy, Peter. "Le traduzioni italiane delle opere letterarie ungheresi." Rivista di studi ungheresi, ns., n.3 (2004): 7-I6. 